\title{
La Città dei Bambini e dei Ragazzi à Gênes, un exemple à suivre
}

Fernande Vedel (fernande.vedel@univ-amu.fr) Professeur émérite, P2IM, UMR7345, Université d’Aix-Marseille, 13397 Marseille Cedex 20

\section{Dans l'ancien port de Gênes} se trouve depuis vingt ans La Città dei Bambini e dei Ragazzi, née d'une collaboration avec la Cité des enfants de La Villette.

Plus de $3000 \mathrm{~m}^{2}$ pour captiver, émerveiller les 2-13 ans par des rencontres actives et variées avec les sciences et les technologies.

Les installations laissent la part belle à l'initiative et à la découverte individuelle dans l'amusement et le jeu, à I'aide d'outils éducatifs et de diffusion scientifique qui privilégient la manipulation, l'observation et l'expérience directe, mais aussi la " gestion » du doute. Toute une stratégie permettant aux enfants d'aller au-delà des acquis scolaires, en proposant une participation active sur des thèmes concrets et attrayants.

Je remercie Anna Gugliandolo pour la photo de la page 31, et Alberto Cappato, directeur de Porto Antico, pour la photo de la page 32.

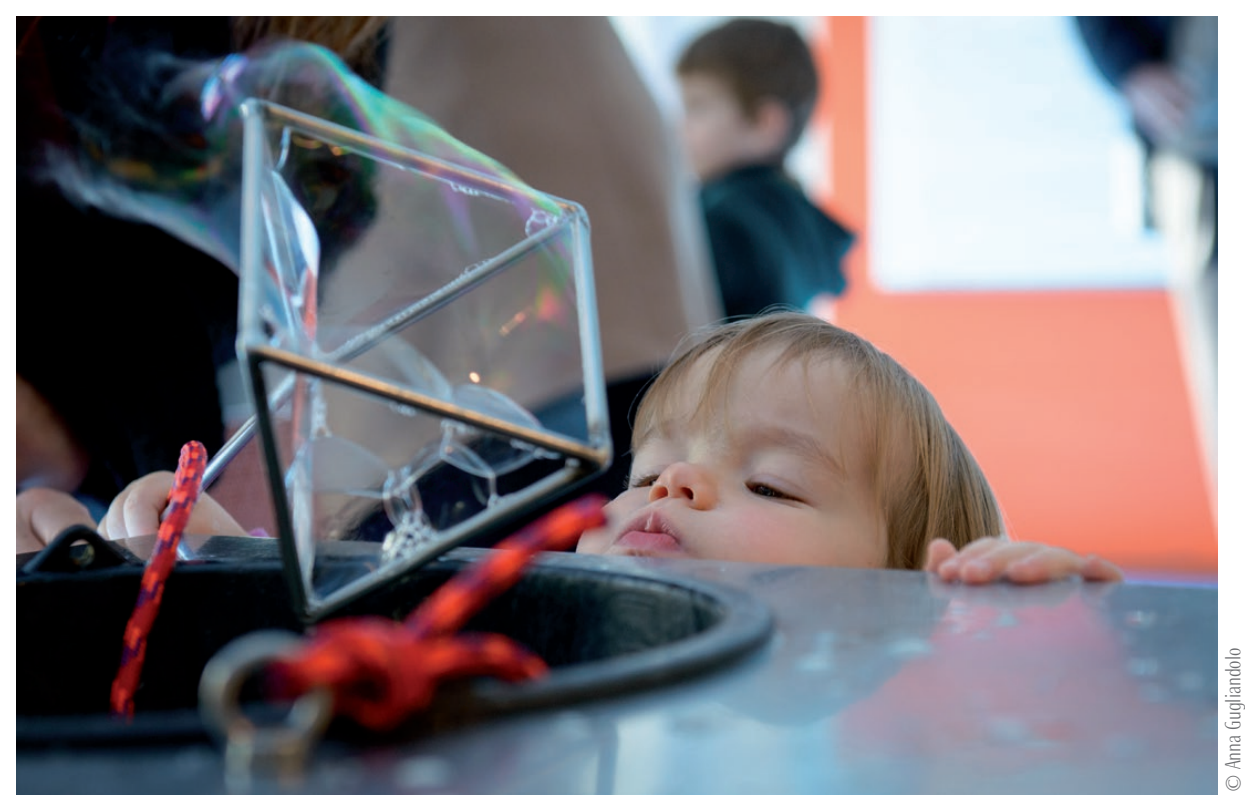

En mélangeant de l'eau et du savon, le tout-petit (2-3 ans) fabrique des bulles de différents types et découvre un monde de formes et de couleurs.

\section{Présentation générale}

La Cité des enfants et des jeunes de Gênes (La Città), lieu de divertissement éducatif au cœur de la science, s'adresse aux enfants et aux adolescents de 2 à 13 ans. Elle est située à proximité de l'Aquarium, plus précisément dans l'ancien entrepôt de coton. Cette partie de l'ancienne zone portuaire fût donnée en concession à Porto Antico S.p.A. (ville, chambre de commerce et port autonome) et aménagée en espace touristique et culturel avec loisirs et restauration.

Les activités culturelles du Port de Gênes sont gérées par la société privée Costa Edutainment. Le personnel de La Città dépend en partie du Consortium Agorà de Ligurie, société qui regroupe dix coopératives sociales de services à la personne. Cette organisation un peu complexe, ne semble pas porter préjudice au développement harmonieux de La Città, qui a la volonté d'associer la découverte scientifique et technologique et le jeu. La Città propose également un solide accompagnement didactique. Créée en 1997, elle attire scolaires, familles et autres groupes grand public (150 000 visiteurs par an, dont 30000 scolaires), et son rôle de diffuseur de culture scientifique est une belle réussite.

La Città propose à ses visiteurs une surface passée récemment de 2000 à $3000 \mathrm{~m}^{2}$ sur un niveau unique et dans un espace largement éclairé par de grandes fenêtres donnant sur le port. L'espace est organisé en secteurs développés spécifiquement par classe d'âge, tout en restant accueillant pour tous. Ces secteurs sont ceints de murets suffisamment hauts pour les délimiter, mais sans cacher leurs " occupants " ni être un obstacle à la circulation.

L'unité du lieu est assurée par un sol bleu comme la Méditerranée qui, associé à 


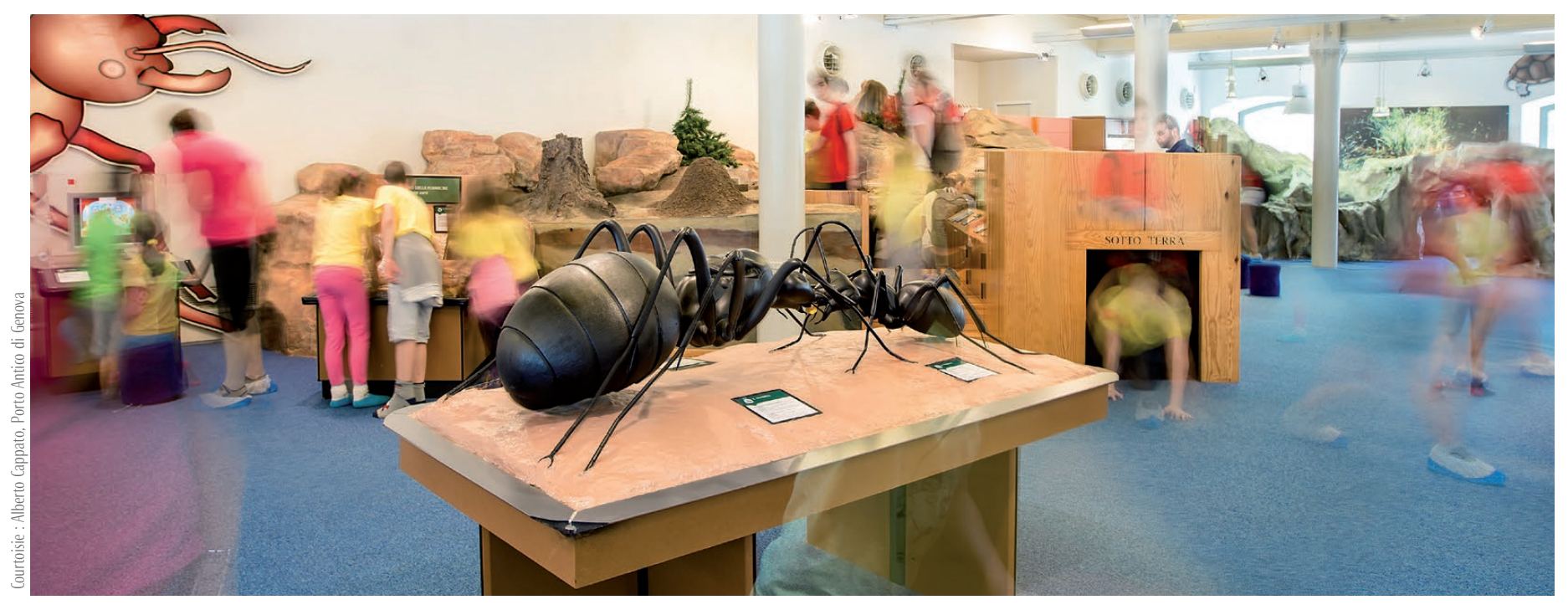

Observation de la fourmilière géante dans l'espace des 6-13 ans.

\section{\>}

l'orange franc des murets et des portails, crée une ambiance dynamique et stimulante où l'on se sent bien et prêt à «toucher " à tout. Ainsi, trois grandes aires accueillent les enfants : une pour les 3-5 ans (niveau maternelle) et deux pour les 6-13 ans (élémentaire et début du collège), complétées par un petit espace pour les 2-3 ans. Chaque thème scientifique, auquel la signalétique associe une couleur, se retrouve dans un "îlot ». Les thèmes abordés sont variés et ciblent les différentes tranches d'âge auxquelles s'adresse La Città. Ils émanent essentiellement de la physique, la chimie, les sciences naturelles, l'environnement, les technologies et les communications. Pour les plus jeunes, c'est la découverte de soi-même par les sens qui est privilégiée. Pour les niveaux "école élémentaire ", ce sera l'observation et l'expérimentation. Enfin, pour les plus grands, l'apprentissage actif des technologies (production d'énergies d'origine renouvelable, fabrication de robots...), en stimulant la créativité pour donner envie d'inventer les métiers qui n'existent pas encore !

\section{Les différents espaces}

\section{L'espace des deux-trois ans}

La zone des tout-petits propose un espace adapté aux découvertes sensorielles et motrices (La perception de mon corps), en stimulant le partage entre l'enfant et l'adulte qui l'accompagne. Dans Le petit bois, le bambino va découvrir et appréhender une petite maison, un miroir, la nature avec un ravin, une tanière, une grotte et même une rivière, de quoi créer aussi des émotions : l'adulte peut à tout moment pénétrer dans l'espace et s'associer au jeu.

\section{L'espace des trois-cinq ans}

Dans la zone des 3-5 ans, trois activités sont proposées : Les mains dans l'eau, Moi et les autres et Le monde qui m'entoure. Les 3-5 ans, équipés de cirés, vont appréhender et s'approprier l'eau en jouant avec des cuves, des barrages, la maitriser avec des pompes de cale ou en testant des jets d'eau capables de soulever des balles. C'est à plusieurs, sur un "vrai " chantier et avec un casque, qu'ils vont apprendre à construire une maison avec des grues, des chariots, des briques et tout ce qui est nécessaire, ou encore découvrir le monde extérieur en éprouvant la force du vent, reconnaître avec le toucher, voire avec des miroirs déformants...

\section{L'espace des six-treize ans}

Pour les 6-13 ans, la démarche concerne essentiellement le vivant, les technologies et la physique. Le monde vivant est abordé par une fourmilière géante construite avec ses éléments de base (dôme, restaurant, cimetière), où l'on examine directement le comportement et l'organisation en société des fourmis rouges; l'observation s'effectue au-dessus et en dessous du sol et à travers un tunnel. Un étang creusé dans l'argile, avec deux bassins séparés abritant deux spécimens de tortues, montre l'importance de l'environnement sur la continuité de l'espèce, la température de l'incubateur déterminant le sexe des nouveau-nés. L'investigation va jusqu'à l'usage de binoculaires et de microscopes électroniques.
WeporTV est un véritable studio de télévision, avec ses parois de verre l'isolant du monde extérieur, qui permet d'apprendre avec des professionnels les secrets de la télévision (mise en page visuelle) et les ficelles du métier, ainsi qu'à se défendre contre les pièges des médias en développant le sens critique. L'émission réalisée est ensuite postée sur le web et diffusée sur la télé locale.

Le Digiwall (mur d'escalade informatisé) combine les caractéristiques d'un vrai mur d'escalade à la technologie des jeux informatiques, associant agilité physique et agilité intellectuelle, où des prises s'éclairent pour former la voie au milieu de sons et de musique.

Des jeux-expériences permettent d'aborder le concept d'énergie, ses différentes formes et ses transformations, ainsi que la production de sources d'énergie renouvelables.

Un jeu d'ombres interactif permet de simuler une cascade de sable. L'utilisation de l'optique met en évidence des effets sur la perception visuelle en perturbant la liaison œil-cerveau : illusions d'optique, miroirs déformants, erreurs de perspective (chambre d'Ames, chaise de Beuchet). Un banc de fabrication de bulles de savon de différentes formes permet de rapprocher physique et mathématiques.

On peut aussi reconstituer l'aventure transatlantique d'un paquebot en jouant, s'initier au Morse, faire des nœuds, découvrir les routes de migration et fabriquer un compas pour calculer l'azimuth.

Au total, un beau programme, avec plus de 90 animations interactives ! 


\section{L'encadrement}

Six animateurs, diplômés universitaires, dont deux en sciences de l'éducation, deux en sciences de l'environnement, un en sciences forestières et un en sciences chimiques, constituent l'équipe d'encadrement. De mars à juin, mois les plus chargés, le Consortium Agorà recrute des étudiants et de jeunes diplômés (de 23 à 35 ans), en sciences humaines pour travailler avec les enfants plus jeunes, et en sciences plus "dures " pour interagir avec les enfants plus âgés.

\section{Conclusion}

La configuration des activités permet de stimuler l'observation, la manipulation, l'expérimentation, mais aussi le doute et le droit à l'erreur en développant une pensée critique et responsable. Les nombreux ateliers favorisent l'apprentissage coopératif, tout en laissant chacun s'exprimer. Le "spectaculaire" (dimensions des expériences, p.ex. les lampes RVB permettent de faire des ombres à taille humaine) est utilisé à bon escient pour frapper et favoriser l'attention et l'observation. L'utilisation des nouvelles technologies sans excès n'interdit pas de présenter par exemple une "exposition " d'appareils de radio plus anciens et de relier l'environnement de tous les jours et son évolution aux technologies plus récentes.

La Città est pilotée par L'Associazione Festival della Scienza, association de promotion, de valorisation et de diffusion de la culture scientifique et technique. Les contenus scientifiques des animations sont enrichis par des partenariats avec les services éducatifs de la ville de Gênes et la Faculté d'architecture de l'université (Industrial Design), et par des collaborations étroites : Cité des enfants (Universcience), Universités de Gênes et de Rome, Conseil National de la Recherche (CNR) italien. La mise en scène est soignée jusqu'à faire intervenir les " grands » du métier, comme la société allemande Hüttinger Interactive Exhibitions.

La Città dei bambini e ragazzi de Gênes, la plus grande d'Italie, est en permanente évolution et reconfiguration. Elle vient d'accueillir, par exemple, un Tinkering Lab (lieu dédié au "faire soi-même "), à l'image de celui de l'Exploratorium de San Francisco. Elle se démarque d'autres musées scientifiques pour la jeunesse par son implantation originale, structurée à la fois suivant l'âge ou plutôt le niveau affectif/scolaire et par thématique ; c'est une des rares à avoir développé une unité pour les tout-petits. L'agencement fait que l'enfant ou le pré-adolescent y trouve ses repères et se sent chez lui, avec peut-être une densité d'écrans moindre qu'à la maison ; on y agit réellement en contact avec la matière et la vie. On n'a pas l'impression, comme à Nemo (Amsterdam) ou même Eureka (San Sebastian), d'être happé par une structure géante. La Città est une unité à part entière, sans être rattachée à un ensemble plus vaste, et cette intimité du lieu favorise l'implication du visiteur.

\section{En savoir plus}

- Guida Doccenti (guide pour les enseignants, en italien), à télécharger à l'adresse : www.portoantico.it/wpcontent/uploads/2016/10/ guida-docenti-cdbr-2016-2017.pdf

- Anna Kaiser, « La città dei bambini e dei ragazzi, in Genova », (en italien, téléchargeable), Studium Educationis, anno XIII - n.2 (juin 2012), pp. 95-106,

\section{Visites organisées}

Des visites sont organisées sur inscription pour les groupes et les scolaires dès la maternelle.

Un large choix est possible avec des objectifs bien définis, adaptés aux différents niveaux (de la maternelle au collège).

La liste des possibilités, accessible dans le « guide de l'enseignant » (Guida Doccenti) téléchargeable, est classée selon les critères : approfondissement thématique, activités, laboratoire (travaux pratiques). L'offre porte, en particulier, sur l'eau et I'air, la créativité liée aux contes de fées, la nutrition, les cinq sens, les facultés sensorielles, l'environnement (voyager avec Darwin, la pollinisation...), la physique (propriétés de l'eau : décantation, canalisation, mélanges...), la chimie (réactions en chaîne), la géométrie (puzzle chinois tangram pour les 3 ans), I'astronomie (étoiles, planètes et leurs secrets). Les animations sont étudiées pour solliciter le sens critique et pour encourager la formulation $d^{\prime}$ hypothèses qui expliquent le pourquoi des phénomènes observés. Les activités sont limitées à $20 \mathrm{mn}$ et la durée des visites à 1 h 20 .

La Città propose également des animations au sein des établissements scolaires.

Dans le cadre de la loi imposant la formation en alternance, La Città accueille chaque année de nombreux étudiants en didactique, elle reçoit les enseignants pour coordonner ses offres avec leur enseignement..

En fin de semaine, La Città dei bambini e dei ragazzi propose à son jeune public des animations spéciales sur des thématiques toujours actuelles, instructives et amusantes.

Chaque année, début décembre, un programme spécial célèbre l'anniversaire de l'institution, qui participe aussi activement au Festival des sciences de Gênes fin octobre.

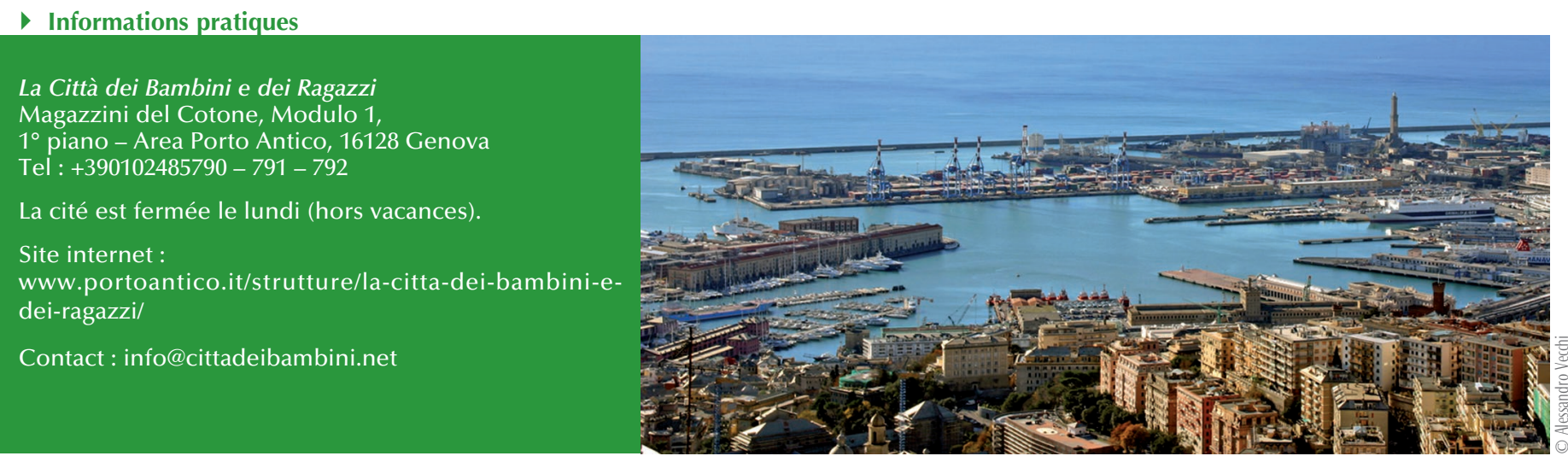

